\title{
RESPUESTA DE FRESA CV. FESTIVAL A LA SALINIDAD
}

\section{RESPONSE OF STRAWBERRY CV. FESTIVAL TO SALINITY}

\author{
Sandra L. González-Jiménez , Ana Ma. Castillo-González*, \\ Ma. del Rosario García-Mateos ${ }^{1}$, Luis A. Valdez-Aguilar², \\ Carmen Ybarra-Moncada ${ }^{3}$ y Edilberto Avitia-García'
}

\begin{abstract}
'Universidad Autónoma Chapingo (UACh), Departamento de Fitotecnia, Chapingo, Estado de México, México. ${ }^{2}$ Universidad Autónoma Agraria Antonio Narro, Departamento de Horticultura, Buenavista, Saltillo, Coahuila, México. ${ }^{3}$ UACh, Instituto de Alimentos, Chapingo, Estado de México, México.
\end{abstract}

*Autor de correspondencia (anasofiacasg@hotmail.com)

\section{RESUMEN}

La fresa (Fragaria $x$ ananassa Duch.) es un cultivo de importancia socioeconómica para México, su fruto tiene un alto valor nutricional para el humano; sin embargo, no se conoce el efecto de la salinidad en los cultivares comerciales; por tal motivo, el objetivo de este trabajo fue conocer el efecto de la salinidad en la solución nutritiva sobre el crecimiento, concentración nutrimental, contenido de prolina, rendimiento y calidad de los frutos de fresa cv. Festival. Se establecieron plantas en macetas de $1.8 \mathrm{~L}$ de capacidad con tezontle (granulometría 2 a $3 \mathrm{~mm}$ ) en un sistema hidropónico abierto con la solución de Steiner. Los tratamientos consistieron en soluciones nutritivas con conductividades eléctricas crecientes: 2.0 (testigo), 2.3, 2.5 y $2.7 \mathrm{dS} \mathrm{m}^{-1}$, que se obtuvieron con la mezcla de $\mathrm{NaCl}$ y sulfatos de $\mathrm{Ca}$ y $\mathrm{Mg}$ en la solución, aplicadas en el riego cada $4 \mathrm{~d}$, a partir de la etapa de floración. El rendimiento y número de frutos por planta no se afectaron. La concentración de prolina en la raíz se incrementó en 56, 111 y 97 \% con las conductividades de 2.3, 2.5 y 2.7, respectivamente. La concentración foliar de $\mathrm{N}, \mathrm{Ca}$, Na y $\mathrm{Cl}$ no se afectó por la salinidad, pero con la CE de 2.7 el contenido de P disminuyó $55 \%$, el de Mg 52 $\%$, mientras que el K aumentó $88 \%$ y el S $117 \%$. No se encontraron diferencias estadísticas en el peso, diámetro, firmeza e índice de redondez del fruto. El contenido de azúcares solubles totales disminuyó 18 \% y la relación sólidos solubles/acidez titulable $11 \%$ con 2.7 de CE. Conforme la CE se elevó, la concentración de fenoles, flavonoides, antocianinas, la actividad antioxidante y el porcentaje de inhibición disminuyó; sólo la vitamina $C$ aumentó. Los aumentos aplicados de CE no fueron lo suficientemente altos para afectar el rendimiento; la salinidad sólo incremento el contenido de vitamina $C$ en los frutos.

Palabras clave: Fragaria $\times$ ananassa Duch., antioxidantes, calidad del fruto, estado nutrimental, prolina, vitamina C.

\section{SUMMARY}

Strawberry (Fragaria $x$ ananassa Duch.) is a crop of socioeconomic importance for Mexico, its fruits have high nutritional value for humans; however, the effect of salinity on commercial cultivars is unknown; for this reason, the objective of this study was to determine the effect of salinity of the nutrient solution on growth, nutrient status, proline content, yield and quality of strawberry fruits cv. Festival. Plants were established in $1.8 \mathrm{~L}$ post capacity filled with volcanic rock (tezontle) (granulometry 2 to $3 \mathrm{~mm}$ ) in an open hydroponic system with Steiner's solution. The treatments consisted of nutrient solutions with increasing electrical conductivities: 2.0 (control), 2.3, 2.5 and $2.7 \mathrm{dS} \mathrm{m}^{-1}$ obtained with the mixture of $\mathrm{NaCl}$ and $\mathrm{Ca}$ and $\mathrm{Mg}$ sulfates in the solutions applied by irrigation every $4 \mathrm{~d}$ starting at the flowering stage.
Yield and number of fruits per plant were not affected. The concentration of proline in the root increased by 56,111 and $97 \%$ with electric conductivities of 2.3, 2.5 and $2.7 \mathrm{dS} \mathrm{m}^{-1}$, respectively. Leaf concentration of $\mathrm{N}, \mathrm{Ca}, \mathrm{Na}$ and $\mathrm{Cl}$ was not affected by increasing salinity, but $\mathrm{P}$ and $\mathrm{Mg}$ decreased by 55 and 52 $\%$, respectively, in plants irrigated with solution of $2.7 \mathrm{dS} \mathrm{m}^{-1}$, while $\mathrm{K}$ increased by $88 \%$ and $S$ by $117 \%$. No statistical differences were found in the weight, diameter, firmness and roundness index of fruit. The total soluble sugar content decreased by $18 \%$ and the total soluble solids/tritable acidity ratio decreased by $11 \%$ with solution of $2.7 \mathrm{dS} \mathrm{m}^{-1}$. As the electrical conductivity increased the concentration of phenols, flavonoids, anthocyanins, antioxidant activity and percentage of inhibition decreased; only vitamin $\mathrm{C}$ increased. The applied increases in electric conductivity were not high enough to affect yield; salinity only increased the vitamin $\mathrm{C}$ content in fruits.

Index words: Fragaria $\times$ ananassa Duch., antioxidants, fruit quality, nutrient status, proline, vitamin C.

\section{INTRODUCCIÓN}

La salinidad es uno de los mayores problemas que afecta a la agricultura mundial, los suelos agrícolas que incrementaron su productividad hasta en $50 \%$ durante el siglo XX, gracias a la adopción de la fertilización química, son ahora suelos con problemas de salinidad (Fageria et al., 2011); una combinación de procesos naturales y malas prácticas agrícolas es la causante de ello (Munns y Tester, 2008). La salinidad es causada por la interacción de varias sales como cloruro de sodio y sulfato de magnesio en zonas costeras; o de bicarbonato de sodio, cloruro de solio y sulfato de magnesio en suelos sódicos (Ríos-Gómez et al., 2010), lo que conduce a altos valores de conductividad eléctrica en el suelo, que tienen un efecto negativo en el crecimiento y rendimiento de la mayoría de las plantas cultivadas. La respuesta de las diferentes especies vegetales ante la salinidad varía considerablemente en función de su genética y tolerancia inherente; sin embargo, la generalidad de los cultivos hortofrutícolas es muy sensible (Goreta et al., 2007). La salinidad afecta de dos formas principales a la planta, una por efecto del daño osmótico, que implica un desabastecimiento de 
agua al interior celular con la consecuente disminución del crecimiento y la otra por la toxicidad que el exceso y desbalance de iones genera en las hojas (Saqib et al., 2006); no obstante, esta disminución del crecimiento vegetativo puede tener algunas consecuencias benéficas en la calidad del fruto. Khayyat et al., 2007 y Keutgen y Keutgen (2003) reportaron una reducción en la producción de frutos de fresa Elsanta y Korona, pero con mejora en la calidad de los frutos con estrés salino de 40 y 80 meq L ${ }^{-1}$ de $\mathrm{NaCl}$, debido al incremento de azúcares reductores y ácidos ocasionado por un menor contenido de agua.

En México el cultivo de la fresa es importante desde el punto de vista socioeconómico, debido a que genera empleo y divisas por ser producto de exportación para su consumo en fresco, congelado y procesado. Los principales estados productores son Michoacán, Baja California, Baja California Sur, Estado de México, Jalisco, Oaxaca y Morelos, con una producción de 471,972 t (SIAP, 2018). Cuando el cultivo crece en condiciones de salinidad, en niveles que exceden su tolerancia, la planta disminuye su tasa de crecimiento, el número de hojas, el área foliar y la producción de frutos (Garriga et al., 2015). El cultivar Festival, obtenido en Florida, Estados Unidos, es de los más utilizados en México, dado que después de varios ciclos ha demostrado su eficiencia en campo; es de fotoperiodo corto, la planta es vigorosa, los frutos tienen buen sabor, color rojo intenso, forma cónica y pulpa firme (USPTO, 2004). Varios autores han evaluado el efecto del estrés salino en especies como frijol mungo (Phaseolus radiatus L.) (Rashid et al., 2004), olivo (Olea europea L.) (Goreta et al., 2007), trigo (Triticum aestivum L.) (Saqib et al., 2006) y algunos cultivares de fresa (Fragaria x ananassa Duch.) (Garriga et al., 2015), cuyas investigaciones se basaron exclusivamente en dosis de $\mathrm{NaCl}$ como único causante de la salinidad; sin embargo, esta situación en los suelos es causada por la presencia de iones de $\mathrm{Ca}^{2+}, \mathrm{Mg}^{2+}, \mathrm{Cl}^{-}, \mathrm{Na}^{+}$, sulfato $\left(\mathrm{SO}_{4}^{2-}\right)$, bicarbonato $\left(\mathrm{HCO}_{3}{ }^{-}\right)$y en algunos casos $\mathrm{K}^{+}$ y $\mathrm{NO}_{3}{ }^{-}$, dependiendo del tipo de suelo (Parihar et al., 2015; Ríos-Gómez et al., 2010).

A pesar de la importancia económica del cultivo de la fresa para México, país que a nivel mundial ocupa el tercer lugar en producción (Saavedra-Romero et al., 2018) y del impacto en la salud humana que tiene el consumo de los frutos, poco se sabe acerca de la tolerancia a la salinidad y de su impacto en la calidad del fruto en los cultivares comerciales más importantes en el país. La presente investigación tuvo como objetivo conocer el efecto de salinidad moderada (2.0 a 2.7 dS $\mathrm{m}^{-1}$ ) en la solución nutritiva sobre el crecimiento, concentración nutrimental, contenido de prolina en la raíz, rendimiento y calidad de los frutos para determinar la factibilidad de producir fresa
Festival en zonas con suelos o agua con salinidad.

\section{MATERIALES Y MÉTODOS}

\section{Sitio experimental}

La investigación se realizó en un invernadero de cristal de la Universidad Autónoma Chapingo, ubicada a los $19^{\circ}$ $20^{\prime}$ latitud norte y $98^{\circ} 53^{\prime}$ longitud oeste a $2240 \mathrm{~m}$ de altitud; la temperatura y humedad relativa durante el ciclo del cultivo (de agosto de 2016 a marzo de 2018) se registró con un data logger HOBO ${ }^{8}$ U12-012 (Burlington, Vermont, EUA); la temperatura media en ese periodo varió de 18 a 23 ${ }^{\circ} \mathrm{C}$ y la humedad relativa de 46 a $63 \%$.

\section{Material biológico y tratamientos}

Se utilizaron plántulas de fresa del cultivar Festival procedentes de Zamora, Michoacán, las que se establecieron en macetas de $1.8 \mathrm{~L}$ de capacidad con tezontle de 2 a $3 \mathrm{~mm}$ de diámetro en un sistema hidropónico abierto. Para la elaboración de las soluciones nutritivas se utilizó agua destilada; también se consideró el balance de aniones y cationes de las relaciones iónicas de Steiner (1984).

Los tratamientos de salinidad, expresados en términos de conductividad eléctrica (CE), fueron: 2.0 (testigo), 2.3, 2.5 y $2.7 \mathrm{dS} \mathrm{m}^{-1}$, la concentración de los iones que integraron las soluciones nutritivas completas se presenta en el Cuadro 1. los micronutrimentos se abastecieron con $0.035 \mathrm{~g} \mathrm{~L}^{-1} \mathrm{de}$ la mezcla TradeCorp ${ }^{\circledR}$ AZ. Las plantas se regaron diaria y manualmente, entre las 7:30 y 8:30 de la mañana, con agua destilada durante las primeras dos semanas después del trasplante (SDT), a las tres SDT se regó con $200 \mathrm{~mL}$ de la solución nutritiva de CE de $2.0 \mathrm{dS} \mathrm{m}^{-1}$ (testigo), a las 12 SDT el volumen se incrementó a $300 \mathrm{~mL}$ y a partir de las 30 SDT éste fue de $500 \mathrm{~mL}$, debido al crecimiento de las plantas. En todos los casos se tuvo un drenaje aproximado del 30 $\%$. Los tratamientos se aplicaron cada $4 \mathrm{~d}$, a partir de las 40 SDT (floración) hasta las 77 SDT ( 9 meses de aplicación de tratamientos), el resto de los días se regaba con la solución testigo (CE de $2.0 \mathrm{dS} \mathrm{m}^{-1}$ ).

El diseño experimental fue completamente al azar con 10 repeticiones; se colocó una planta por maceta la cual se consideró como la unidad experimental; sin embargo, para las determinaciones de las variables destructivas (excepto para el análisis nutrimental, descrito posteriormente), con la finalidad de contar con suficiente material biológico se tomó un grupo de tres plantas como unidad experimental, y el número de repeticiones utilizado fue de tres. 
Cuadro 1. Concentración de iones de las soluciones nutritivas salinas utilizadas en el cultivo de fresa cv. Festival en Chapingo, México.

\begin{tabular}{|c|c|c|c|c|c|c|c|c|c|c|c|}
\hline \multirow{2}{*}{ 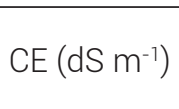 } & $\mathrm{NO}_{3}^{-}$ & $\mathrm{H}_{2} \mathrm{PO}_{4}^{-2}$ & $\mathrm{SO}_{4}^{-2}$ & $\mathrm{HCO}_{3}^{-}$ & $\mathrm{Cl}^{-}$ & $\mathrm{NH}_{4}^{+}$ & $\mathrm{K}^{+}$ & $\mathrm{Ca}^{2+}$ & $\mathrm{Mg}^{2+}$ & $\mathrm{Na}^{+}$ & \multirow{2}{*}{$\mathrm{pH}$} \\
\hline & \multicolumn{10}{|c|}{$\left(\mathrm{mmol} \mathrm{L}^{-1}\right)$} & \\
\hline 2.0 & 15.76 & 1.00 & 0.82 & 1.00 & 0.00 & 0.87 & 6.97 & 4.00 & 0.60 & 1.00 & 5.9 \\
\hline 2.3 & 15.76 & 1.00 & 1.29 & 1.00 & 1.36 & 0.87 & 6.97 & 4.70 & 0.79 & 2.36 & 5.9 \\
\hline 2.5 & 15.76 & 1.00 & 1.96 & 1.00 & 2.04 & 0.87 & 6.97 & 5.05 & 1.11 & 3.04 & 5.9 \\
\hline 2.7 & 15.76 & 1.00 & 2.62 & 1.00 & 2.73 & 0.87 & 6.97 & 5.40 & 1.42 & 3.73 & 5.9 \\
\hline
\end{tabular}

\section{Variables evaluadas}

Al final del experimento, 77 SDT, se evaluaron las variables siguientes:

Rendimiento y número de frutos por planta. Se cosecharon, cuantificaron y pesaron la totalidad de los frutos por unidad experimental.

Peso fresco y seco de planta. Se seleccionaron al azar tres plantas por tratamiento, cada planta se seccionó en hojas y corona con raíz, se registró el peso fresco y seco por separado con una balanza digital OHAUS ${ }^{\circledR}$ modelo Scout Pro (Parsippany-Troy Hills, New Jersey, EUA); para el secado se usó una estufa con aire forzado BINDERß (Tuttlingen, Alemania) a $65^{\circ} \mathrm{C}$ por $72 \mathrm{~h}$ o hasta peso constante.

Análisis nutrimental. Se formó una muestra compuesta, integrada por dos plantas por tratamiento, las que se separaron en hojas (con sus peciolos) y corona con raíz. Dicha muestra compuesta se dividió en tres partes iguales y cada parte representó una repetición. Se determinó la concentración de N, P, K, Ca, Mg, S, Na y Cl. La determinación de $\mathrm{N}$ se realizó por el método de micro Kjeldahl y la de los demás elementos por espectrometría de emisión atómica de inducción con plasma acoplado ICP modelo 725-ES (Agilent ${ }^{\circledR}$, Santa Clara, California, EUA); en todos los casos se siguió la metodología descrita por Alcántar y Sandoval (1999).

Prolina. Se determinó con el método de Bates et al. (1973); de tres plantas por tratamiento, se pesaron $0.5 \mathrm{~g}$ de muestra fresca de ápices de raíz. Las lecturas se realizaron a $520 \mathrm{~nm}$ en un espectrofotómetro Thermo Scientific $\AA$ modelo Genesys 10 UV (New York, EUA). La concentración de prolina se estimó a partir de una curva patrón de 0 a 39 $\mu \mathrm{g} \mathrm{mL}^{-1}$ de prolina Sigma Aldrich®. Todo lo anterior se hizo por triplicado.

Durante el periodo de fructificación se cosecharon todos los frutos de todas las repeticiones cuando éstos alcanzaron el color rojo brillante y se evaluaron las variables siguientes:

Peso fresco. Se registró en g en la totalidad de frutos por tratamiento, con una balanza digital OHAUS® modelo Scout Pro (Parsippany-Troy Hills, New Jersey, EUA).

Índice de redondez. En todos los frutos de la unidad experimental se midió la longitud y diámetro ecuatorial en $\mathrm{mm}$ con un vernier digital General ${ }^{\circledR}$ No. 143 (Suiza), con estos datos se calculó la relación longitud/diámetro, con la que se obtuvo el índice de redondez, donde los valores menores que 1.0 son considerados como ovalados, mayores que 1.0 como alargados y valores de $1.0 \mathrm{como}$ redondos (Martínez-Bolaños et al., 2008).

Firmeza del fruto. Se evaluó en los mismos frutos utilizados para el índice de redondez, con un penetrómetro Qa Supplies ${ }^{\circledR}$ modelo FT O2 (Norfolk, Virginia, EUA) con punta de $2.3 \mathrm{~mm}$ de grosor a la altura del diámetro ecuatorial. Los resultados se expresaron en newtons $(\mathrm{N})$.

Sólidos solubles totales (SST). Se registró en ${ }^{\circ}$ Brix con un refractómetro digital Atago ${ }^{\circledR}$ modelo PAL-1 (Saitama, Japón) en la totalidad de los frutos.

Acidez titulable (AT). Se utilizó la técnica descrita por la AOAC (1980); el porcentaje de acidez se calculó con base en el ácido cítrico, que es el ácido que se encuentra en mayor cantidad en los frutos de fresa. Con los datos de SST y AT se calculó la relación SST/AT.

Azúcares solubles totales (AST). Se determinaron con el método de antrona descrito por Witham et al. (1971). Las lecturas se realizaron a $600 \mathrm{~nm}$ en un espectrofotómetro Thermo Spectronic ${ }^{\circledR}$ modelo Genesys 10 UV (New York, EUA). La concentración de azúcares se estimó a partir de una curva patrón que contenía hasta $250 \mu \mathrm{g}$ de glucosa $\mathrm{mL}^{-1}$. 


\section{Determinación de compuestos antioxidantes en frutos}

Extracto metanólico. En un mortero de porcelana se trituraron $3 \mathrm{~g}$ de pulpa de fresa (obtenida de la mezcla de 10 frutos por tratamiento) con $30 \mathrm{~mL}$ de metanol acuoso 80 \% (v/v), la mezcla se homogeneizó con agitación en un vórtex; posteriormente, se colocó en sonicación por 15 min a temperatura ambiente, la mezcla se dejó reposar por 24 h y se centrifugó por 10 min a 1,400 × g. Con este extracto se cuantificaron los compuestos fenólicos (Chang et al., 2002).

Fenoles. Se tomaron $250 \mu \mathrm{L}$ del sobrenadante del extracto metanólico previamente preparado, se agregaron $250 \mu \mathrm{L}$ del reactivo Folin-Ciocalteu $0.2 \mathrm{~N}$ y $2 \mathrm{~mL}$ de una solución $0.7 \mathrm{M} \mathrm{de} \mathrm{Na}_{2} \mathrm{CO}_{3}$, la mezcla se incubó por $2 \mathrm{~h}$ a temperatura ambiente y en oscuridad. Se registró la absorbancia a 765 nm en un espectrofotómetro Thermo Spectronic $\AA$ modelo Genesys 10 UV (New York, EUA). La concentración se calculó a partir de una curva patrón preparada con ácido gálico y se expresó en mg equivalentes de ácido gálico por $100 \mathrm{~g}$ de peso fresco (mg EAG $\cdot 100 \mathrm{~g}^{-1}$ p.f.) de acuerdo con el método de Waterman y Mole (1994).

Flavonoides. Se tomaron $500 \mu \mathrm{L}$ del extracto metanólico obtenido previamente, se agregaron $1.5 \mathrm{~mL}$ de etanol 95 $\%, 100 \mu \mathrm{L}$ de solución $\mathrm{AlCl}_{3} 10 \%, 100 \mu \mathrm{L}$ de $\mathrm{CH}_{3} \mathrm{COOK} 1$ M y $2.8 \mathrm{~mL}$ de agua destilada; la mezcla se incubó por 30 min a temperatura ambiente. La absorbancia se leyó a $415 \mathrm{~nm}$ en un espectrofotómetro Thermo Spectronic ${ }^{\circledR}$ modelo Genesys 10 UV (New York, EUA). La concentración se determinó con una curva patrón de flavonol quercetina (Chang et al., 2002). Los resultados se expresaron en mg equivalentes de quercetina por $100 \mathrm{~g}$ de peso fresco ( $\mathrm{mg}$ EQ $100 \mathrm{~g}^{-1}$ p.f.).

Antocianinas. Se prepararon dos tubos de ensayo (tubo 1 y tubo 2) con $200 \mu \mathrm{L}$ del extracto metanólico previamente obtenido; al tubo 1 se le adicionaron $1.8 \mathrm{~mL}$ de una solución amortiguadora $(\mathrm{HCl} / \mathrm{KCl})$ de $\mathrm{pH}=1$, al tubo 2 se le agregaron $1.8 \mathrm{~mL}$ de una solución amortiguadora $\left(\mathrm{CH} 3 \mathrm{COOH} / \mathrm{CH} 3 \mathrm{COONa} \cdot 3 \mathrm{H}_{2} \mathrm{O}\right)$ de $\mathrm{pH}=4.5$. Se leyó la absorbancia de ambas mezclas a 510 y $700 \mathrm{~nm}$ con un espectrofotómetro Thermo Spectronic ${ }^{\circledR}$ modelo Genesys 10 UV (New York, EUA). La absorbancia total (At) se calculó de la siguiente manera:

$$
A_{t}=\left[\left(A_{510}-A_{700}\right)_{p H=1.0}\right]-\left[\left(A_{510}-A_{700}\right)_{p H=4.5}\right]
$$

La concentración de antocianinas (AN) se calculó de la siguiente manera:

$$
\mathrm{AN}=\mathrm{A}_{\mathrm{t}} \times \mathrm{PM} \times \mathrm{FD} \times 1000 /(\varepsilon \times 1)
$$

donde: $A_{t}$ es la absorbancia total, PM es el peso molecular (449.2 $\mathrm{g} \mathrm{mol}^{-1}$ ) del estándar cianidina 3-glucósido, FD es el factor de dilución y $\varepsilon$ es la absortividad molar del estándar $(26,900)$. La concentración se expresó en mg de antocianinas por $100 \mathrm{~g}$ de peso fresco, según lo descrito por Giusti y Wrolstad (2001).

Contenido de vitamina C. Se determinó con el método 967.21 de la AOAC (1995); se pesaron $0.5 \mathrm{~g}$ de muestra fresca, los que se maceraron con $5 \mathrm{~mL}$ de ácido metafosfórico $1 \%$, se dejaron reposar por 45 min; posteriormente, se filtró y aforó a $5 \mathrm{~mL}$. Del extracto se tomó $1 \mathrm{~mL}$ y se diluyó en 9 $\mathrm{mL}$ de dicloroindofenol $\left(12 \mathrm{mg} \mathrm{L}^{-1}\right)$. Se leyó la absorbancia a $515 \mathrm{~nm}$ con un espectrofotómetro Thermo Spectronic ${ }^{\circledR}$ modelo Genesys 10 UV (New York, EUA), se utilizó agua desionizada como blanco.

Actividad antioxidante del fruto. Se determinó con el método ABTS descrito por Re et al. (1999). La determinación se hizo con una curva estándar con trólox (ácido 6-hidroxi2,5,7,8-tetrametilcroman-2-carboxílico). Los resultados se expresaron en $\mu \mathrm{mol}$ equivalentes de trólox por $\mathrm{g}$ de peso fresco. Para calcular el porcentaje de inhibición del radical libre $\mathrm{ABTS}^{+}$se empleó la fórmula:

$$
\text { \% de inhibición }=\left[\left(A_{l}-A_{F}\right) / A_{l}\right] \times 100
$$

donde: $A_{l}$ es la absorbancia inicial del radical libre y $A_{F}$ es la absorbancia final de la reacción con la muestra.

\section{Análisis estadístico}

Se realizó análisis de varianza y se aplicó la prueba de medias de Tukey $(P \leq 0.05)$ con el programa SAS versión 9 (SAS Institute, 2002).

\section{RESULTADOS Y DISCUSIÓN}

\section{Respuesta de la planta y rendimiento}

El peso fresco y seco total, el de las hojas y de la raíz de las plantas disminuyó con las conductividades de 2.3 a 2.7 (Cuadro 2); esto es debido a la inhibición del crecimiento por el estrés hídrico que sufre la planta, causado por el exceso de sales disueltas en la solución externa, lo que limita la absorción de agua por las raíces (Parihar et al., 2015).

El rendimiento y número de frutos por planta no se afectaron por el incremento en la CE (Cuadro 3), a pesar de que la salinidad es uno de los factores ambientales que afecta la producción de los cultivos, como se observó en fresa Camarosa y en los genotipos chilenos Bau y Cucao sujetos a 30 y $60 \mathrm{mM}$ de $\mathrm{NaCl}$ (Garriga et al., 2015); en 
Cuadro 2. Efecto de la CE en la solución nutritiva sobre el peso fresco y seco de la raíz y parte aérea de plantas de fresa cv. Festival bajo condiciones de invernadero.

\begin{tabular}{lcccccc}
\hline \multirow{2}{*}{ CE $\left(\mathrm{dS} \mathrm{m}^{-1}\right)$} & PFT & PST & PFH & PSH & PFR & PSR \\
\cline { 2 - 7 } & \multicolumn{7}{c}{$(\mathrm{g})$} \\
\hline 2.0 & $90.80 \pm 3.45 \mathrm{a}^{+}$ & $15.10 \pm 0.8 \mathrm{a}$ & $38.94 \pm 1.20 \mathrm{a}$ & $7.61 \pm 0.29 \mathrm{a}$ & $51.86 \pm 2.30 \mathrm{a}$ & $7.49 \pm 0.55 \mathrm{a}$ \\
2.3 & $62.44 \pm 2.42 \mathrm{~b}$ & $10.86 \pm 0.3 \mathrm{~b}$ & $24.12 \pm 1.95 \mathrm{~b}$ & $4.81 \pm 0.31 \mathrm{~b}$ & $38.32 \pm 1.50 \mathrm{~b}$ & $6.05 \pm 0.16 \mathrm{~b}$ \\
2.5 & $64.32 \pm 2.73 \mathrm{~b}$ & $11.07 \pm 0.6 \mathrm{~b}$ & $24.46 \pm 1.90 \mathrm{~b}$ & $4.96 \pm 0.30 \mathrm{~b}$ & $39.86 \pm 0.96 \mathrm{~b}$ & $6.10 \pm 0.32 \mathrm{ab}$ \\
2.7 & $55.78 \pm 4.25 \mathrm{~b}$ & $9.50 \pm 0.6 \mathrm{~b}$ & $18.38 \pm 1.57 \mathrm{~b}$ & $3.80 \pm 0.40 \mathrm{~b}$ & $37.40 \pm 2.70 \mathrm{~b}$ & $5.69 \pm 0.23 \mathrm{~b}$ \\
CV (\%) & 10.7 & 12.1 & 14.28 & 14.17 & 10.80 & 12.48 \\
DSH & 13.33 & 2.55 & 6.84 & 1.35 & 8.18 & 1.43 \\
\hline
\end{tabular}

${ }^{\top}$ Medias \pm error estándar. Letras distintas en las columnas son estadísticamente diferentes (Tukey, P $\left.\leq 0.05\right)$. CV: coeficiente de variación, PFT: peso fresco total, PST: peso seco total, PFH: peso fresco de hojas, PSH: peso seco de hojas, PFR: peso fresco de raíz, PSR: peso seco de raíz, DSH: diferencia significativa honesta.

Cuadro 3. Rendimiento, número de frutos y concentración de prolina en fresa cv. Festival cultivada bajo diferentes niveles de CE en la solución nutritiva.

\begin{tabular}{lccc}
\hline $\mathrm{CE}\left(\mathrm{dS} \mathrm{m} \mathrm{m}^{-1}\right)$ & Rendimiento $\left(\mathrm{g} \mathrm{planta}^{-1}\right)$ & Número de frutos por planta & Prolina en raíz $\left(\mathrm{mg} \mathrm{mL}^{-1}\right)$ \\
\hline 2.0 & $91.15 \pm 10.54 \mathrm{a}^{\dagger}$ & $11.7 \pm 0.95 \mathrm{a}$ & $7.90 \pm 0.49 \mathrm{c}$ \\
2.3 & $93.41 \pm 11.97 \mathrm{a}$ & $12.0 \pm 1.54 \mathrm{a}$ & $12.30 \pm 0.03 \mathrm{~b}$ \\
2.5 & $94.47 \pm 13.05 \mathrm{a}$ & $13.2 \pm 1.76 \mathrm{a}$ & $16.62 \pm 0.18 \mathrm{a}$ \\
2.7 & $111.97 \pm 15.50 \mathrm{a}$ & $13.5 \pm 1.86 \mathrm{a}$ & $15.54 \pm 0.66 \mathrm{a}$ \\
$\mathrm{CV}(\%)$ & 41.73 & 39.77 & 9.17 \\
DSH & 49.13 & 6.03 & 1.53 \\
\hline
\end{tabular}

${ }^{\dagger}$ Medias \pm error estándar. Letras distintas en las columnas son diferentes estadísticamente (Tukey, P $\left.\leq 0.05\right)$. CV: coeficiente de variación, DSH: diferencia significativa honesta.

este trabajo, con el cv. Festival, los incrementos en las conductividades fueron tan pequeños que no afectaron su producción.

La concentración de prolina en la raíz se incrementó en 56,111 y $97 \%$ con las conductividades de $2.3,2.5$ y 2.7 $\mathrm{dS} \mathrm{m}^{-1}$, respectivamente (Cuadro 3 ). Resultados similares se observaron en el cv. Camarosa y los genotipos chilenos Bau y Cucao (Garriga et al., 2015) y en los cultivares Camarosa y Chandler (Turhan y Eris, 2009) en respuesta a la salinidad. La acumulación de prolina en las hojas y en la raíz normalmente ocurre bajo situaciones de estrés salino, debido a que esta molécula es un osmoprotector que contribuye al ajuste osmótico del citoplasma celular, ayuda a mantener el contenido hídrico y a estabilizar las estructuras subcelulares como membranas y proteínas y permite que se mantenga sin alteración el potencial redox de las células (Parihar et al., 2015).

La concentración foliar de N, Ca, Na y Cl no se vio afectada por los tratamientos; con la CE de 2.7 la concentración de P disminuyó en $55 \%$ y la de $\mathrm{Mg}$ en $52 \%$, en tanto que la de $\mathrm{K}$ se incrementó en 88 \% y la de S en $117 \%$ en comparación con el control. En la raíz sólo se afectó la concentración de S; el más alto valor se registró con la CE de 2.5 (Cuadro 4).

Parihar et al. (2015) mencionan que la salinidad por $\mathrm{NaCl}$ provoca desbalances nutrimentales en el medio que rodea a la raíz y en la planta, debido a que afecta la disponibilidad y absorción de algunos nutrimentos; bajo estas condiciones, la planta presenta una disminución en la concentración de N, por la interacción antagónica que existe entre el $\mathrm{Na}$ y el $\mathrm{NH}_{4}$ y entre el $\mathrm{Cl}$ y el $\mathrm{NO}_{3}$, como se observó en aguacatero (Persea americana Mill.) y cítricos por Bar et al. (1997). Así mismo, la absorción de P, K, Ca y Mg también disminuye por los antagonismos entre el $\mathrm{Cl}$ y los fosfatos y la insolubilidad de las sales fosfatadas por el elevado pH que se presenta en el medio salino; el Na por su parte, presenta una interacción negativa con K, Ca y Mg, lo que conduce a la menor absorción de estos elementos (Parihar et al., 2015).

En el presente estudio con el cv. Festival se observó una importante disminución en la absorción de P por la mayor absorción de $\mathrm{S}$ (sulfatos), elementos que presentan una interacción antagónica (Fageria et al., 2011). El Na y el Cl 
no afectaron la absorción de N, Ca y K debido a que los tratamientos de salinidad se conformaron con la adición de $\mathrm{NaCl}$ más los sulfatos de Ca y Mg, como se muestra en el Cuadro 1; por lo que estos dos iones no estuvieron en la solución nutritiva en una concentración tal que afectara la absorción de dichos nutrimentos; incluso, la concentración

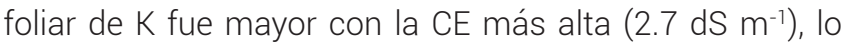
que sugiere una absorción eficiente de $\mathrm{K}$ en las plantas de fresa; esta situación también la observaron Keutgen y Pawelzik (2009) en los cultivares de fresa Korona y Elsanta cultivados con 40 y $80 \mathrm{mmol} \mathrm{L}^{-1}$ de $\mathrm{NaCl}$ en la solución nutritiva; en contraste, en olivo (Olea europea L.) cv. Leccino y Oblica, la concentración de $\mathrm{K}$ disminuyó conforme la salinidad aumentó de 66 a $166 \mathrm{mM}$ de $\mathrm{NaCl}$ (Goreta et al., 2007), lo que indica que la respuesta nutrimental ante la salinidad varía entre las especies.

\section{Calidad del fruto}

No se encontraron diferencias estadísticas entre tratamientos en el peso fresco, diámetro, firmeza e índice de redondez; la longitud del fruto fue mayor en $6.6 \%$ con respecto al testigo, con las conductividades de 2.3 y 2.7 , pero sin diferencia con la de $2.5 \mathrm{dS} \mathrm{m}^{-1}$ (Cuadro 5). El contenido de azúcares solubles totales (AST) disminuyó $18 \%$ con la máxima CE (2.7 dS $\left.\mathrm{m}^{-1}\right)$ en comparación con el testigo; también, la relación sólidos solubles totales/acidez titulable (SST/AT) fue menor con 2.7 de CE (Cuadro 6). De acuerdo con Hancock (1999), el valor óptimo de SST para frutos rojos está entre 6 y $9 \%$, valores que se alcanzaron en esta investigación en los frutos de todos los tratamientos. El porcentaje de acidez titulable (0.8\%) fue el adecuado en todos los niveles de salinidad evaluados, ya que Dale y Luby (1991) indican como intervalo óptimo de 0.42 a 1.24 $\%$ en fresa. La relación SST/AT adecuada es de 8.5 a 14 (Keutgen y Pawelzik, 2007), por lo que los frutos de Festival presentaron una relación adecuada, con valores de 9 a 10 en todos los tratamientos (Cuadro 6). El contenido de AST (de 5.6 a $7 \mathrm{~g}$ por $100 \mathrm{~g}$ de p.f) también se encontró en el intervalo adecuado, según Dale y Luby (1991), quienes establecieron los valores de 4.1 a $6.6 \mathrm{~g}$ como los mejores para fresa. Los resultados encontrados coinciden con los observados en el cv. comercial Camarosa (sensible a salinidad) y los ecotipos chilenos Bau y Cucao (menos sensibles) cultivados con 30 y $60 \mathrm{mM} \mathrm{NaCl}$ en la solución nutritiva, en los cuales no se afectó el peso, tamaño, firmeza ni SST de los frutos; sólo la AT se incrementó en los ecotipos chilenos (Garriga et al., 2015).

La concentración de fenoles, flavonoides y antocianinas disminuyó con el aumento de la CE; en contraste, la concentración de vitamina C aumentó conforme la CE fue

Cuadro 4. Concentración nutrimental (\%) en hojas y raíces de fresa cv. Festival cultivado bajo diferentes niveles de conductividad eléctrica (CE) en la solución nutritiva en condiciones de invernadero.

\begin{tabular}{lcccccccc}
\hline CE $\left(\mathrm{dS} \mathrm{m} \mathrm{m}^{-1}\right)$ & $\mathrm{N}$ & $\mathrm{P}$ & $\mathrm{K}$ & $\mathrm{Ca}$ & $\mathrm{Mg}$ & $\mathrm{S}$ & $\mathrm{Na}$ & $\mathrm{Cl}$ \\
\hline 2.0 & $2.84 \pm 0.00 \mathrm{a}^{+}$ & $0.40 \pm 0.04 \mathrm{a}$ & $1.11 \pm 0.09 \mathrm{~b}$ & $1.12 \pm 0.04 \mathrm{a}$ & $0.46 \pm 0.00 \mathrm{a}$ & $0.12 \pm 0.00 \mathrm{~b}$ & $0.23 \pm 0.01 \mathrm{a}$ & $0.021 \pm 0.00 \mathrm{a}$ \\
2.3 & $2.80 \pm 0.02 \mathrm{a}$ & $0.44 \pm 0.02 \mathrm{a}$ & $0.95 \pm 0.02 \mathrm{~b}$ & $1.37 \pm 0.14 \mathrm{a}$ & $0.49 \pm 0.00 \mathrm{a}$ & $0.23 \pm 0.03 \mathrm{ab}$ & $0.24 \pm 0.02 \mathrm{a}$ & $0.039 \pm 0.00 \mathrm{a}$ \\
2.5 & $2.56 \pm 0.28 \mathrm{a}$ & $0.38 \pm 0.03 \mathrm{a}$ & $1.03 \pm 0.06 \mathrm{~b}$ & $1.19 \pm 0.20 \mathrm{a}$ & $0.37 \pm 0.03 \mathrm{a}$ & $0.21 \pm 0.00 \mathrm{ab}$ & $0.33 \pm 0.01 \mathrm{a}$ & $0.035 \pm 0.00 \mathrm{a}$ \\
2.7 & $3.29 \pm 0.10 \mathrm{a}$ & $0.18 \pm 0.00 \mathrm{~b}$ & $2.09 \pm 0.31 \mathrm{a}$ & $0.72 \pm 0.01 \mathrm{a}$ & $0.22 \pm 0.02 \mathrm{~b}$ & $0.26 \pm 0.00 \mathrm{a}$ & $0.31 \pm 0.02 \mathrm{a}$ & $0.037 \pm 0.00 \mathrm{a}$ \\
$\mathrm{CV}(\%)$ & 9.04 & 13.42 & 18.38 & 16.52 & 8.80 & 12.74 & 13.05 & 15.74 \\
$\mathrm{DSH}$ & 1.05 & 0.19 & 0.97 & 0.73 & 0.13 & 0.10 & 0.14 & 0.02 \\
& & & & $R a i ́ z$ & & & & \\
2.0 & $2.29 \pm 0.22 \mathrm{a}$ & $0.26 \pm 0.00 \mathrm{a}$ & $0.54 \pm 0.11 \mathrm{a}$ & $1.97 \pm 0.27 \mathrm{a}$ & $0.51 \pm 0.00 \mathrm{a}$ & $0.22 \pm 0.00 \mathrm{~b}$ & $0.39 \pm 0.04 \mathrm{a}$ & $0.042 \pm 0.00 \mathrm{a}$ \\
2.3 & $1.96 \pm 0.16 \mathrm{a}$ & $0.30 \pm 0.03 \mathrm{a}$ & $0.44 \pm 0.04 \mathrm{a}$ & $2.49 \pm 0.00 \mathrm{a}$ & $0.41 \pm 0.04 \mathrm{a}$ & $0.24 \pm 0.01 \mathrm{ab}$ & $0.36 \pm 0.04 \mathrm{a}$ & $0.045 \pm 0.00 \mathrm{a}$ \\
2.5 & $1.85 \pm 0.20 \mathrm{a}$ & $0.36 \pm 0.00 \mathrm{a}$ & $0.46 \pm 0.12 \mathrm{a}$ & $2.53 \pm 0.02 \mathrm{a}$ & $0.69 \pm 0.21 \mathrm{a}$ & $0.28 \pm 0.00 \mathrm{a}$ & $0.48 \pm 0.00 \mathrm{a}$ & $0.051 \pm 0.00 \mathrm{a}$ \\
2.7 & $2.18 \pm 0.15 \mathrm{a}$ & $0.34 \pm 0.03 \mathrm{a}$ & $0.51 \pm 0.00 \mathrm{a}$ & $2.33 \pm 0.17 \mathrm{a}$ & $0.41 \pm 0.00 \mathrm{a}$ & $0.26 \pm 0.00 \mathrm{ab}$ & $0.37 \pm 0.04 \mathrm{a}$ & $0.039 \pm 0.00 \mathrm{a}$ \\
$\mathrm{CV}(\%)$ & 13.26 & 11.97 & 26.29 & 12.53 & 31.96 & 4.37 & 15.45 & 15.34 \\
DSH & 1.11 & 0.15 & 0.52 & 1.18 & 0.65 & 0.04 & 0.25 & 0.02
\end{tabular}

${ }^{\dagger}$ Medias \pm error estándar. Letras distintas en las columnas son estadísticamente diferentes dentro de cada estructura (Tukey, P $\left.\leq 0.05\right)$. CV: coeficiente de variación, DSH: diferencia significativa honesta. 
mayor, de tal manera que el mayor valor $\left(32.37 \mathrm{mg} \mathrm{g}^{-1} \mathrm{de}\right.$ p.f.) se registró con $2.7 \mathrm{dS} \mathrm{m}^{-1}$. La actividad antioxidante y, en consecuencia, el porcentaje de inhibición disminuyó con la salinidad (Cuadro 7). Estos resultados no coinciden con los reportados para otros cultivos como tomate (Solanum lycopersicum L.), donde el número total de frutos comerciales no fue afectado por la CE de 2.2, 3.5 y 4.5 dS $\mathrm{m}^{-1}$, pero con esos valores de CE el contenido de licopeno, vitamina $\mathrm{C}$, fenoles totales y actividad antioxidante se incrementó significativamente (Moya et al., 2017).

En los frutos de fresa cv. Camarosa y el ecotipo chileno Bau la concentración de antocianinas tendió a disminuir con el incremento de la salinidad dada por $\mathrm{NaCl}$ a 30 y 60 mM (Garriga et al., 2015), como sucedió también en los frutos de Festival evaluados en esta investigación; sin embargo, en los cultivares de fresa Elsanta (sensible) y Korona (menos sensible), tratados con 40 y $80 \mathrm{mmol} \mathrm{L}^{-1}$ de $\mathrm{NaCl}$ en la solución nutritiva, se observó incrementó en la capacidad antioxidante, contenido de fenoles y antocianinas conforme aumentó la salinidad, mientras que la concentración de ácido ascórbico disminuyó (Keutgen y Pawelzik, 2007), lo cual refleja la variabilidad en la respuesta a la salinidad entre los cultivares de fresa.
El estrés por salinidad en las plantas induce al incremento en la generación de especies reactivas de oxígeno (estrés oxidativo), las cuales causan daño a los lípidos de las membranas, proteínas y ácidos nucleicos, con una consecuente reducción en la productividad de los cultivos (Parihar et al., 2015). Ante este daño, las plantas han desarrollado un sistema protector con capacidad antioxidante mediante la síntesis de algunos compuestos, el cual varía entre las especies; entre estos compuestos se encuentran las antocianinas, flavonoides, fenoles y vitamina C (Zhu, 2001; Munns y Tester, 2008). En este caso, en los frutos del cv. Festival obtenidos bajo condiciones de salinidad la capacidad antioxidante sólo se expresó con la acumulación de vitamina $\mathrm{C}$, lo que pudo disminuir el efecto del estrés oxidativo, causado por la acumulación de especies reactivas de oxígeno que se presenta bajo condiciones de salinidad

\section{CONCLUSIONES}

La salinidad moderada (CE de 2.0 a $2.7 \mathrm{dS} \mathrm{m}^{-1}$ ) aplicada en fresa cv. Festival cada $4 \mathrm{~d}$ a partir de la etapa de floración provoca disminución en la acumulación del peso

Cuadro 5. Efecto de la CE de la solución nutritiva en las características físicas de los frutos de fresa cv. Festival bajo condiciones de invernadero.

\begin{tabular}{lccccc}
\hline C.E. $\left(\mathrm{dS} \mathrm{m} \mathrm{m}^{-1}\right)$ & Peso $(\mathrm{g})$ & Longitud $(\mathrm{mm})$ & Diámetro $(\mathrm{mm})$ & Índice de redondez & Firmeza $(\mathrm{N})$ \\
\hline 2.0 & $8.01 \pm 0.30 \mathrm{a}^{+}$ & $29.70 \pm 0.55 \mathrm{~b}$ & $25.03 \pm 0.32 \mathrm{a}$ & $1.23 \pm 0.05 \mathrm{a}$ & $2.28 \pm 0.06 \mathrm{a}$ \\
2.3 & $8.51 \pm 0.34 \mathrm{a}$ & $31.65 \pm 0.60 \mathrm{a}$ & $24.99 \pm 0.32 \mathrm{a}$ & $1.26 \pm 0.01 \mathrm{a}$ & $2.30 \pm 0.06 \mathrm{a}$ \\
2.5 & $7.74 \pm 0.26 \mathrm{a}$ & $30.31 \pm 0.53 \mathrm{ab}$ & $24.44 \pm 0.25 \mathrm{a}$ & $1.24 \pm 0.01 \mathrm{a}$ & $2.25 \pm 0.07 \mathrm{a}$ \\
2.7 & $8.51 \pm 0.27 \mathrm{a}$ & $31.67 \pm 0.47 \mathrm{a}$ & $25.10 \pm 0.26 \mathrm{a}$ & $1.27 \pm 0.01 \mathrm{a}$ & $2.30 \pm 0.07 \mathrm{a}$ \\
CV $(\%)$ & 34.96 & 17.02 & 11.28 & 24.02 & 29.67 \\
DSH & 20.53 & 1.90 & 1.01 & 0.09 & 1.12
\end{tabular}

${ }^{\top}$ Medias \pm error estándar. Letras distintas en las columnas son diferentes estadísticamente (Tukey, P $\left.\leq 0.05\right)$. CV: coeficiente de variación, DSH: diferencia significativa honesta.

Cuadro 6. Efecto de la conductividad eléctrica de la solución nutritiva en las características químicas de frutos de fresa cv. Festival bajo condiciones de invernadero.

\begin{tabular}{lcccc}
\hline CE $\left(\mathrm{dS} \mathrm{m}^{-1}\right)$ & AST $\left(\mathrm{g} 100 \mathrm{~g}^{-1}\right.$ p.f. $)$ & SST $(\%)$ & AT $(\%)$ & Relación SST/AT \\
\hline 2.0 & $6.84 \pm 0.30 \mathrm{a}^{+}$ & $8.5 \pm 0.18 \mathrm{a}$ & $0.824 \pm 0.01 \mathrm{a}$ & $10.52 \pm 0.25 \mathrm{a}$ \\
2.3 & $6.80 \pm 0.27 \mathrm{a}$ & $8.46 \pm 0.20 \mathrm{a}$ & $0.824 \pm 0.01 \mathrm{a}$ & $10.41 \pm 0.29 \mathrm{a}$ \\
2.5 & $7.17 \pm 0.32 \mathrm{a}$ & $8.35 \pm 0.16 \mathrm{a}$ & $0.827 \pm 0.02 \mathrm{a}$ & $10.16 \pm 0.26 \mathrm{ab}$ \\
2.7 & $5.60 \pm 0.23 \mathrm{~b}$ & $7.96 \pm 0.17 \mathrm{a}$ & $0.882 \pm 0.02 \mathrm{a}$ & $9.32 \pm 0.34 \mathrm{~b}$ \\
CV (\%) & 14.55 & 19.8 & 14.60 & 20.10 \\
DSH & 1.04 & 0.59 & 0.06 & 1.05
\end{tabular}

${ }^{\top}$ Medias \pm error estándar. Letras distintas en las columnas son diferentes estadísticamente (Tukey, P $\left.\leq 0.05\right)$. CE: conductividad eléctrica, AST: azúcares solubles totales, SST: sólidos solubles totales, AT: acidez titulable, CV: coeficiente de variación, DSH: diferencia significativa honesta. 
fresco y seco de la planta. La CE más alta (2.7) disminuye la concentración foliar de P y Mg en $50 \%$, pero provoca aumento del K en 88 \% y del S en $117 \%$ : La concentración de $\mathrm{N}, \mathrm{Ca}, \mathrm{Na}$ y $\mathrm{Cl}$ no se afecta por ninguno de los valores evaluados de CE. Los tratamientos de salinidad no afectaron el rendimiento ni los parámetros comerciales de calidad del fruto como peso, índice de redondez, firmeza, sólidos solubles totales y acidez titulable; en tanto que el contenido de compuestos antioxidantes como fenoles, flavonoides, antocianinas y actividad antioxidante disminuyó conforme aumentó la CE, pero la vitamina $\mathrm{C}$ se incrementó hasta en $34 \%$ con la CE más alta. El contenido de prolina en la raíz se elevó hasta en 100 \% con la CE de 2.7, como mecanismo de respuesta y protección del estrés hídrico.

\section{BIBLIOGRAFÍA}

Alcántar G. G. y M. Sandoval V. (1999) Manual de Análisis Químico de Tejido Vegetal: Guía de Muestreo, Preparación, Análisis e Interpretación. Sociedad Mexicana de la Ciencia del Suelo. Chapingo, Estado de México. 156 p.

AOAC, Association of Official Analytical Chemists (1980) Official Methods of Analysis. 12th ed. Association of Official Analytical Chemists. Washington, D.C. USA. 1018 p.

AOAC, Association of Official Analytical Chemists (1995) Official Methods of Analysis. 16th ed. Association of Official Analytical Chemists. Washington, D.C. USA. 2490 p.

Bar Y., A. Apelbaum, U. Kafkafı and R. Goren (1997) Relationship between chloride and nitrate and its effect on growth and mineral composition ofavocadoand citrusplants. JournalofPlantNutrition 20:715-731, https://doi.org/10.1080/01904169709365288

Bates L., R. P. Waldren and I. D. Teare (1973) Rapid determination of free proline for water-stress studies. Plant and Soil 39:205-207, https://doi.org/10.1007/BF00018060

Chang C. C., M. H. Yang, H. M. Wen and J. C. Chern (2002) Estimation of total flavonoid content in propolis by two complementary colorimetric methods. Journal of Food and Drug Analysis 10:178-182.

Dale A. and J. J. Luby (1991) The Strawberry into the 21st Century. Timber Press. Portland, Oregon, USA. $292 \mathrm{p}$.

Fageria N. K., H. R. Gheyi and A. Moreira (2011) Nutrient bioavailability in salt affected soils. Journal of Plant Nutrition 34:945-962, https://doi.org/10.1080/01904167.2011.555578

Garriga M., C. A. Muñoz, P. D. S. Caligari and J. B. Retamales (2015) Effect of salt stress on genotypes of commercial (Fragaria $x$ ananassa) and Chilean strawberry (F. chiloensis). Scientia Horticulturae 195:37-47, https://doi.org/10.1016/j.scienta.2015.08.036

Giusti M. M. and R. E. Wrolstad (2001) Characterization and measurement of anthocyanins by UV-visible spectroscopy. Current Protocols in Food Analytical Chemistry 00:F1.2.1-F1.2.13, https://doi.org/10.1002/0471142913.faf0102s00

Goreta S., V. Bučević-Popović, M. Pavela-Vrančić and S. Perica (2007) Salinity-induced changes in growth, superoxide dismutase activity, and ion content of two olive cultivars. Journal of Plant Nutrition and Soil Science 170:398-403, https://doi.org/10.1002/jpln.200625188

Hancock J. F. (1999) Strawberries. CABI Publishing. New York, USA. 237 p.

Keutgen A. J. and N. Keutgen (2003) Influence of $\mathrm{NaCl}$ salinity stress on fruit quality in strawberry. Acta Horticulturae 609:155-157, https://doi.org/10.17660/ActaHortic.2003.609.20

Keutgen A. and E.Pawelzik(2007)Modifications of taste-relevant compounds in strawberry fruit under $\mathrm{NaCl}$ salinity. Food Chemistry 105:14871494, https://doi.org/10.1016/j.foodchem.2007.05.033

Keutgen A. J. and E. Pawelzik (2009) Impacts of $\mathrm{NaCl}$ stress on plant growth and mineral nutrient assimilation in two cultivars of strawberry. Environmental and Experimental Botany 65:170176, https://doi.org/10.1016/j.envexpbot.2008.08.002

Khayyat M., E. Tafazoli, S. Eshghi, M. Rahemi and S. Rajaee (2007) Salinity, supplementary calcium and potassium effects on fruit yield and quality of strawberry (Fragaria ananassa Duch.). AmericanEurasian Journal of Agricultural and Environmental Sciences 2:539-544.

Martínez-Bolaños M., D. Nieto-Angel, D. Téliz-Ortiz, J. Rodríguez-Alcazar, M. T. Martínez-Damian, H. Vaquera-Huerta y 0. Carrillo-Mendoza (2008) Comparación cualitativa de fresas (Fragaria x ananassa Duch.) de cultivares mexicanos y estadounidenses. Revista Chapingo Serie Horticultura 14:113-119.

Moya C., E. Oyanedel, G. Verdugo, M. F. Flores, M. Urrestarazu and J. E. Álvaro (2017) Increased electrical conductivity in nutrient solution management enhances dietary and organoleptic qualities in soilless culture tomato. HortScience 52:868-872, https://doi. org/10.21273/HORTSCI12026-17

Munns R. and M. Tester (2008) Mechanisms of salinity tolerance. Annual Review of Plant Biology 59:651-681, https://doi.org/10.1146/ annurev.arplant.59.032607.092911

Parihar P., S. Singh, R. Singh, V. P. Singh and S. M. Prasad (2015) Effect of salinity stress on plants and its tolerance strategies: a review. Environmental Science and Pollution Research 22:4056-4075 https://doi.org/10.1007/s11356-014-3739-1

Rashid P., J. L. Karmoker, S. Chakrabortty and B. C. Sarker (2004) The effect of salinity on ion accumulation and anatomical attributes in mungbean (Phaseolus radiatus L. Cv. Bari-3) seedlings International Journal of Agriculture and Biology 6:495-498.

Re R., N. Pellegrini, A. Proteggente, A. Pannala, M. Yang and C. Rice-Evans (1999) Antioxidant activity applying an improved ABTS radical cation decolorization assay. Free Radical Biology and Medicine 26:12311237, https://doi.org/10.1016/S0891-5849(98)00315-3

Ríos-Gómez R., C. E. Salas-García, A. Monroy-Ata and E. Solano (2010) Salinity effect on Prosopis laevigata seedlings. Terra Lationamericana 28:99-107.

Saavedra-Romero L. del C., S. Aranda-Ocampo, D. L. Ochoa-Martínez y A. A. Lira-Vargas (2018) Caracterización fenotípica y genotípica de Xanthomonas fragariae, agente causal de la mancha angular de la fresa en México. Revista Fitotecnia Mexicana 41: 81-85.

Saqib M., C. Zörb and S. Schubert (2006) Salt-resistant and salt-sensitive wheat genotypes show similar biochemical reaction at protein level in the first phase of salt stress. Journal of Plant Nutrition and Soil Science 169:542-548, https://doi.org/10.1002/jpln.200520557

SAS Institute (2002) SAS/STAT. User's Guide. Release 9.0. SAS Institute Inc., Cary, North Carolina. USA. 4424 p.

SIAP, Servicio de Información Agroalimentaria y Pesquera (2018) Anuario estadístico de la producción agrícola. Servicio de Información Agroalimentaria y Pesquera, Secretaría de Agricultura y Desarrollo Rural. Ciudad de México. http://infosiap.siap.gob. mx:8080/agricola_siap_gobmx/AvanceNacionalCultivo.do. (Agosto 2019).

Steiner A. A. (1984) The universal nutrient solution. In: Proceedings of the Sixth International Congress on Soilless Culture. 29 April - 5 May 2004. Secretariat of the International Society for Soilless Culture. Lunteren, The Netherlands. pp:633-649.

Turhan E. and A. Eris (2009) Changes of growth, amino acids, and ionic composition in strawberry plants under salt stress conditions. Communications in Soil Science and Plant Analysis 40:33083322, https://doi.org/10.1080/00103620903325927

USPTO, United States Patent and Trademark Office (2004) 'Strawberry Festival' U. S. Patent PP14,739 P2. United States Patent and Trademark Office. Alexandria, VA, USA. https://patentimages. storage.googleapis.com/69/ae/e3/403581d25e9b64/ USPP14739.pdf (February 2019).

Waterman P. G. and S. Mole (1994) Analysis of Phenolic Plant Metabolites. Blackwell Scientific Publications. Oxford, .K. 238 p.

Witham F. H., D. F. Blaydes and R. M. Devlin (1971) Experiments in Plant Physiology. Van Nostrand Reinhold Company. New York, USA. $245 \mathrm{p}$

Zhu J. K. (2001) Plant salt tolerance. Trends in Plant Science 6:66-71, https://doi.org/10.1016/S1360-1385(00)01838-0 